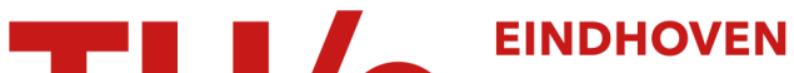 \\ UNIVERSITY OF \\ TECHNOLOGY
}

\section{Speckle-initialized dynamic segmentation of the prostate}

\section{Citation for published version (APA):}

Besseling, R. M. H., Zinger, S., Wijkstra, H., Hendrikx, A. J. M., Hilbers, P. A. J., \& Mischi, M. (2009). Speckleinitialized dynamic segmentation of the prostate. In Proceedings of the 31st Annual International Conference of the IEEE Engineering in Medicine and Biology Society, EMBC 2009, 2-6 September 2009. Minneapolis, Minnesota (pp. 6352-6355). Institute of Electrical and Electronics Engineers.

https://doi.org/10.1109/IEMBS.2009.5333266

DOI:

10.1109/IEMBS.2009.5333266

Document status and date:

Published: 01/01/2009

\section{Document Version:}

Publisher's PDF, also known as Version of Record (includes final page, issue and volume numbers)

\section{Please check the document version of this publication:}

- A submitted manuscript is the version of the article upon submission and before peer-review. There can be important differences between the submitted version and the official published version of record. People interested in the research are advised to contact the author for the final version of the publication, or visit the $\mathrm{DOI}$ to the publisher's website.

- The final author version and the galley proof are versions of the publication after peer review.

- The final published version features the final layout of the paper including the volume, issue and page numbers.

Link to publication

\section{General rights}

Copyright and moral rights for the publications made accessible in the public portal are retained by the authors and/or other copyright owners and it is a condition of accessing publications that users recognise and abide by the legal requirements associated with these rights.

- Users may download and print one copy of any publication from the public portal for the purpose of private study or research.

- You may not further distribute the material or use it for any profit-making activity or commercial gain

- You may freely distribute the URL identifying the publication in the public portal.

If the publication is distributed under the terms of Article 25fa of the Dutch Copyright Act, indicated by the "Taverne" license above, please follow below link for the End User Agreement:

www.tue.nl/taverne

Take down policy

If you believe that this document breaches copyright please contact us at:

openaccess@tue.nl

providing details and we will investigate your claim. 


\title{
Speckle-initialized dynamic segmentation of the prostate
}

\author{
R. M. H. Besseling, S. Zinger, H. Wijkstra, A. J. M. Hendrikx, P. A. J. Hilbers, M. Mischi
}

\begin{abstract}
Echography is a commonly used modality for prostate imaging. Prostate segmentation is the first step in analyzing echographic prostate images. Because of the nature of these images, traditional local image processing operators are inadequate for finding the prostate boundary. Most automated segmentations described in literature require user interaction for contour initializing or editing [1]. Also shape templates are applied as prior knowledge [1]. In this paper, an automatic segmentation method is presented, based on prostate specific image granulation and image intensity. First, a granulation detector is used to extract granulation. Subsequently, the Hessian is adopted to evaluate granulation shape and intensity for the extraction of the prostate-specific dot pattern. This dot pattern is used to construct the contour initialization. A smooth contour model (discrete dynamic contour; DDC) is evolved from this initialization to the final contour. The guiding vector field for the DDC deformation is the gradient vector flow field calculated from an edge map of the original image. The scale of the relevant edges (large compared to granulation) is estimated from the prostate-specific dot pattern. Comparison of automated segmentations with clinical expert manual segmentations reveals a mean sensitivity and accuracy of 0.90 and 0.93 , respectively.
\end{abstract}

\section{INTRODUCTION}

Prostate cancer is the most prevalent cancer and the second highest cause of cancer death in Western men [2] [3] [4] [5]. At present, physical examination (digital rectal examination, DRE) and blood analysis (prostate specific antigen, PSA) are the only non invasive ways to raise prostate cancer suspicion [3] [4]. Since only the dorsal part of the prostate can be investigated by DRE and since also benign prostate hypertrophy increases PSA levels, the diagnosis needs to be confirmed by distributed biopsies.

It is highly desirable to develop an imaging technique for prostate cancer detection. This would allow early diagnosis in a non invasive way and the risk for misdiagnosis (sample error) would be reduced. Easy treatment follow-up and more conservative surgery (instead of radical prostatectomy) would also become possible. Transrectal ultrasound (TRUS) is a useful modality for prostate imaging: it is cost efficient, offers high spatial and temporal resolution and is already used in clinical practice for biopsy guiding and prostate volume estimation. Moreover, improvements have been made recently in ultrasound contrast agents (UCA's; microbubbles) and indicator dilution theory might offer a way to extract

R. M. H. Besseling, S. Zinger, P. A. J. Hilbers and M. Mischi are with the Eindhoven University of Technology, the Netherlands.

H. Wijkstra is with the Department of Urology, Academic Medical Center, University of Amsterdam, the Netherlands.

A. J. M. Hendrikx is with the Department of Urology, Catharina Hospital, Eindhoven, the Netherlands.

Contact: r.m.h.besseling@student.tue.nl local perfusion properties from dynamic contrast enhanced echography of the prostate. These perfusion properties can indicate the presence of cancer related microvascular growth.

Prostate segmentation would be useful both to define the region of interest (ROI) for indicator dilution curve analysis and to estimate the prostate volume. Unfortunately, manual prostate segmentation is a tedious task and an automated approach is desirable.

Echographic contrast is based on tiny differences in acoustic impedance. As a consequence, echographic images are very noisy (speckle) and difficult to segment. Dedicated prostate segmentation methods include edge based approaches using prostate shape models to put the initial contour near relevant edges [1]. Also semiautomatic approaches have been proposed which enhance the image edges and require the user to connect the relevant ones [6]. Other semiautomatic approaches require (partial) initialization of the contour or contour editing [7]. Also statistical approaches have been proposed. Among these are several Bayesian approaches [1] and Gabor filtering, which extracts prostate features from the image and fits a template on these in a multiscale approach. Furthermore, methods which fit shape templates on prostate specific granulation have been investigated [6] and work has been done to construct smooth contours using level set evolution or discrete dynamic contours (DDC) in order to deal with missing edges [1].

Echographic speckle patterns are related to tissue morphology. In this paper, an algorithm is proposed that detects prostate specific granulation to initialize a DDC. The DDC is evolved to a final segmentation under edge guidance. This approach combines the good prostate localization of speckle based methods with the high edge accuracy of edge detectors, in a fully automatic segmentation.

\section{DATA ACQUISITION}

TRUS data was obtained with informed consent from several subjects referred for radical prostatectomy at the Academic Medical Center in Amsterdam (Netherlands). Data was acquired using a clinical ultrasound scanner (Philips iU22) equipped with a transrectal $\mathrm{C} 8-4 \mathrm{v}$ probe at $4 \mathrm{Mhz}$.

In this paper, prostate segmentation from single frames recorded in fundamental mode is discussed. For certain applications (e.g. indicator dilution curve analysis), dynamic data is needed. Resegmentation and/or contour tracking would enable following the contour in time.

\section{Prostate SEGMEntation}

The proposed algorithm for prostate segmentation can be divided in two main parts, shown schematically in Fig. 1. 


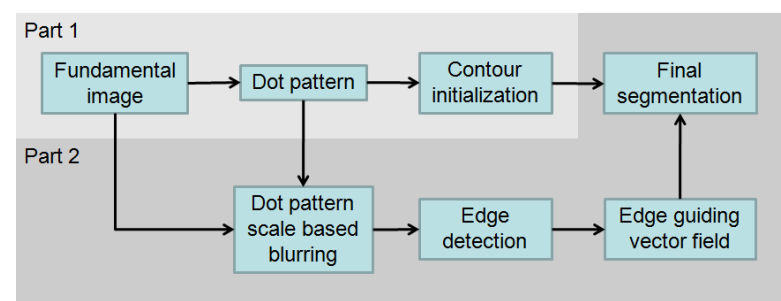

Fig. 1. Segmentation block scheme.

Since the prostate is a gland, it has a typical glandular morphology, which in ultrasound results in a regular dotpattern.

Part 1 of the algorithm is dedicated to extracting the granulation from a fundamental prostate image and to distinguish prostate dots from non-prostate granulation based on shape and intensity. Based on the prostate dots, a contour is initialized.

In part 2 of the algorithm the spacing of the prostate dot pattern is used. The original image is blurred to such an extent that granulation is blurred out. In the resulting image only large scale edges including (parts of) the prostate contour remain. An edge guiding vector field is calculated from this blurred image and used to evolve a DDC, which takes the initial contour as a starting point, to a final contour. The DDC model has a certain smoothness that enables it to bridge regions in which there is no proper edge guiding and thus solves the problem of missing parts of the prostate contour in the original image.

\section{PART 1: CONTOUR INITIALIZATION}

\section{A. Preprocessing}

Preprocessing steps are taken to enhance the granulation of the prostate image [6].

First, median filtering with a $5 \times 5$ window is applied to the original image $f(x, y)$. This is about the granulation size. This new image, which is cleaned of impulsive noise, is referred to as $f_{m f}(x, y)$.

Subsequently, $f_{m f}(x, y)$ is tophat transformed using a $18 \times 18$ octagon structuring element (several times the granulation size). Tophat filtering removes image fluctuations smaller than the structuring element size. By subtracting the tophat filtered image $f_{m f, t h}(x, y)$ from the original image $f(x, y)$, intensity fluctuations of low spatial frequency are removed (Fig. 2).
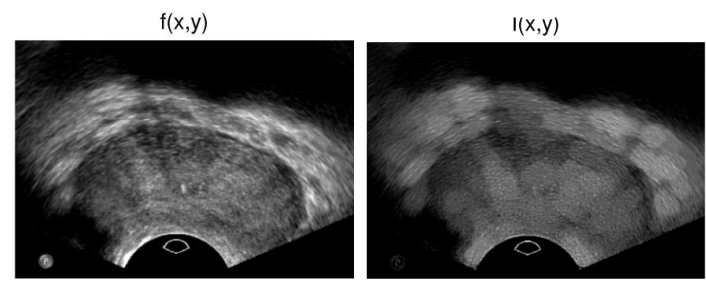

Fig. 2. Original prostate image $f(x, y)$ and preprocessed version $I(x, y)$.

\section{B. Difference of Gaussians}

In [8] the difference of Gaussian (DoG) kernel is proposed to detect granulation of a certain size. The DoG is defined as the difference between two (unnormalized) Gaussians and given by

$$
\operatorname{DoG}_{\sigma, \gamma}(x, y)=\frac{A_{c}}{\gamma} e^{-\frac{x^{2}+y^{2}}{2 \gamma^{2} \sigma^{2}}}-A_{s} e^{-\frac{x^{2}+y^{2}}{2 \sigma^{2}}},
$$

in which $A_{c}$ and $A_{s}$ are constants related to the (narrow) center Gaussian and the (broad) surround Gaussian, respectively. The variance of the center Gaussian is a factor $\gamma^{2}$ smaller than the variance of the surround Gaussian. In literature, it is customary to take $\gamma=0.5$.

The DoG can be used as a detector for granulation that fits the center Gaussian. In order to avoid the undesired negative flanks where granulation is detected, only the positive part of the convolution of the DoG with the preprocessed image $I(x, y)$ is taken. This is given as

$$
u_{\sigma, \gamma}=\left[I * \operatorname{DoG}_{\sigma, \gamma}\right]^{+},
$$

in which, for convenience, the position coordinates $(x, y)$ are omitted. The same is done in the rest of this paper.

$u_{\sigma, \gamma}$ is an image in which granulation is enhanced depending on its local contrast. Since in the investigated images the prostate area is darker than the surrounding tissue, prostate granulation can specifically be enhanced by normalizing the dot image $u_{\sigma, \gamma}$ by the local image intensity, which is given by

$$
s_{\sigma}=I * G_{\sigma},
$$

in which $G_{\sigma}$ is a normalized Gaussian with standard deviation $\sigma$ : the standard deviation of the surround Gaussian. The intensity normalized image is now given as

$$
v_{\sigma, \gamma}=\frac{u_{\sigma, \gamma}}{C s_{\sigma}+1},
$$

in which $C$ is a constant representing the degree of normalization. $C$ is taken equal to $\frac{9}{\max \left(s_{\sigma}\right)}$; the maximal suppression is therefore a factor 10.

\section{Hessian dot evaluation}

The DoG granulation detector enhances dots as well as line segments of the appropriate width, whereas we are only interested in the dots in the prostate region.

The second order differential structure of an image gives important information about dot-like and line-like structures [9]. In order to evaluate the local dot shape of $v_{\sigma, \gamma}$, a Hessian matrix is constructed at each pixel position $(x, y)$ :

$$
H\left(v_{\sigma, \gamma}\right)=\left[\begin{array}{ll}
\frac{\partial^{2} v_{\sigma, \gamma}}{\partial x^{2}} & \frac{\partial^{2} v_{\sigma, \gamma}}{\partial x y} \\
\frac{\partial^{2} v_{\sigma, \gamma}}{\partial x y} & \frac{\partial^{2} v_{\sigma, \gamma}}{\partial y^{2}}
\end{array}\right] .
$$

These second order derivatives must be calculated on the right scale. As an example, $\frac{\partial^{2} v_{\sigma, \gamma}}{\partial x y}$ is calculated as

$$
\begin{aligned}
\frac{\partial^{2} v_{\sigma, \gamma}}{\partial x y} & =\frac{\partial^{2}}{\partial x y}\left(v_{\sigma, \gamma} * G_{\gamma \sigma}\right) \\
& =\frac{\partial^{2} G_{\gamma \sigma}}{\partial x y} * v_{\sigma, \gamma},
\end{aligned}
$$




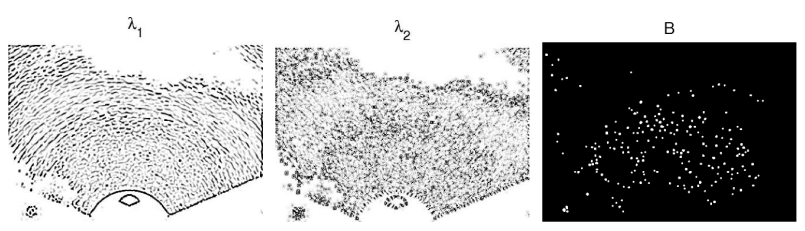

Fig. 3. Image $\lambda_{1}$ highlights pixels which are part of elongated structures and image $\lambda_{2}$ shows round structures. Image $B$ is a thresholded version of masked $I^{\prime}$. Note the granulation in the eigenvalue images is dark because only negative eigenvalues are depicted.

in which the convolution after the first equal sign makes sure the derivative is only sensitive to gradients of the scale of $G_{\gamma \sigma}$ : the scale of the center Gaussian of (1), which matches the dot size. The second equality holds because of the linearity of the operators and permits an efficient implementation.

Since $H\left(v_{\sigma, \gamma}\right)$ is symmetric, its eigenvalue decomposition is always defined. If the two eigenvalues are given by $\lambda_{1}$ and $\lambda_{2}$, for which $\left|\lambda_{1}\right|>\left|\lambda_{2}\right|$, and the two corresponding eigenvectors by $e_{1}$ and $e_{2}$, respectively, the eigenvector $e_{1}$ will point in the longitudinal direction of the local granulation and $\lambda_{1}$ will indicate how strong the granulation in this direction is. Since $e_{1} \perp e_{2}, \lambda_{2}$ gives the granulation extent in the direction of the short axis of the granulation (Fig. 3). We now define the ratio $q$ as

$$
q=\frac{\left|\lambda_{2}\right|}{\left|\lambda_{1}\right|}
$$

For (round) dots $\left|\lambda_{1}\right| \approx\left|\lambda_{2}\right|$ and $q$ approaches 1; for line-like structures $\left|\lambda_{1}\right|>\left|\lambda_{2}\right|$ and $0<q<1$.

Since the second order derivatives of a light dot on a dark background are negative, we are only interested in negative eigenvalues.

Not only the granulation shape can be derived from $\lambda_{1}$ and $\lambda_{2}$, but also the granulation intensity. We define the product $s$ as

$$
s=\left|\lambda_{1}\right|\left|\lambda_{2}\right|,
$$

which quantifies the intensity of the granulation.

Finally, we combine $q$ and $s$ into $I^{\prime}$ as

$$
I^{\prime}=q s .
$$

$I^{\prime}$ is the prostate dots image, where only bright and round structures are left.

In regions where the intensity image $s_{\sigma}$ is dark, weak dots of $u_{\sigma, \gamma}$ are erroneously enhanced by intensity normalization (4). This can seriously affect the derivation of $I^{\prime}$, showing bright dots in wrong places. In order to prevent this, $I^{\prime}$ is set to zero for the lowest $1 \%$ of intensity values.

\section{Contour initialization}

$I^{\prime}$ is converted into a binary image $B$ by thresholding: only dots of at least 0.1 times maximum intensity are kept (Fig. 3 ). On this binary image, a distance transform is performed. If the dot pattern is regular, the most occurring value in the distance transform will be half the characteristic dot-to-dot distance. We can therefore estimate half of the dot spacing distance $r_{c}$ as

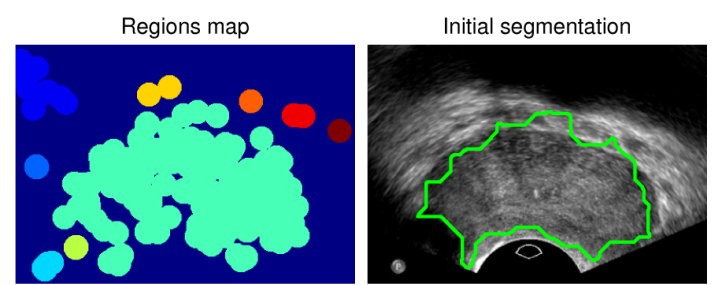

Fig. 4. Interconnected regions and smoothed contour of the largest region, which is taken as prostate contour initialization.

$$
r_{c}=\operatorname{median}(d(B)),
$$

in which $d($.$) stands for the distance transform. If morpho-$ logical dilation is applied to $B$ with a circular kernel of radius $r_{c}$, the dots of the pattern are connected (Fig. 4).

The largest interconnected region is then taken. Morphological closing with a circular kernel or radius $r_{c}$ is performed to fill indentations and holes. Finally, the region is eroded with the same kernel, so that the region rim coincides with the outermost dots. The region rim is taken as prostate contour initialization (Fig. 4) for the following optimization.

\section{PARt 2: DDC EVOlution to Final CONTOUR}

\section{A. Discrete dynamic contour model}

The DDC model is described in [10] and consists of $N$ vertices $\underline{p}_{i}=\left[x_{i}, y_{i}\right], i \in 1,2, \ldots, N$, interconnected by edges. It is a deformable contour whose shape is influenced by internal and external forces.

The internal forces can be described by the related internal energy as

$$
E_{\text {int }}=\sum_{i=1}^{N} \alpha\left|\nabla \underline{p}_{i}\right|^{2}+\beta\left|\nabla^{2} \underline{p}_{i}\right|^{2},
$$

where $\nabla \underline{p}_{i}$ and $\nabla^{2} \underline{p}_{i}$ are the first and second spatial derivative of $p_{i}$, respectively. $\alpha$ is referred to as the elasticity term and $\beta$ is referred to as the rigidity term. In our datset $\alpha=0.03$ and $\beta=0.1$ give satisfactory results.

External forces are calculated from the image and can be used to pull the DDC to certain image regions. Once the desired image locations are put at low energy, the image forces that guide to these minima are given by

$$
\underline{F}_{\mathrm{im}}=-\nabla E_{\mathrm{im}},
$$

in which $E_{\text {im }}$ is the image energy field.

Note from (11) and (12) that the final position of the DDC is a trade off between optimal contour geometry (as compact and smooth as possible) and minimization of the image energy field.

\section{B. Gradient vector field}

$\underline{F}_{\text {im }}$ should be a vector field pointing towards the image edges. We could define $\underline{F}_{\mathrm{im}}=|\nabla f|$, but because of the speckle, strong gradients are found throughout image $f$ and not only at the prostate contour. The scale of the gradient operator could again be controlled by Gaussian blurring as 

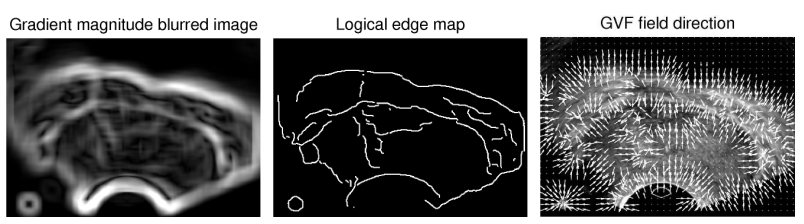

Fig. 5. Blurred image gradient magnitude $\left|\nabla f * G_{r_{c}}\right|$, binary edge map $e$, and normalized GVF field $\frac{u}{\mid \underline{\mid u}}$, calculated from $e$, overlaid on the original image $f$.

in $E_{\mathrm{im}}=\left|\nabla f * G_{r_{c}}\right|$, but since prostate edges are relatively weak (Fig. 5), this is an unreliable approach.

A solution is offered by the gradient vector flow (GVF) field in [10]. The GVF vector field $\underline{u}=\underline{u}(x, y)=$ $[u(x, y), v(x, y)]$ can be seen as the equilibrium solution to the following vector diffusion equations [11],

$$
\begin{aligned}
\underline{u}(x, y, t) & =\mu \nabla^{2} \underline{u}-|\nabla e|^{2}(\underline{u}-\nabla e), \\
\underline{u}(x, y, 0) & =\nabla e,
\end{aligned}
$$

in which $e$ is an edge map and $\underline{u}$ is seen as a function of space and time, so an estimate of $\underline{u}$ can be found iteratively; we use 80 iterations. For $e$ we take the binary edge map of the blurred image $f * G_{r_{c}}$ as given by the Canny edge detector (Fig. 5). As a result, all edges are equally strong. The parameter $\mu$ makes sure that $\underline{u}$ varies smoothly so a pull towards the edges is also present far from them. This is fundamentally different from $\nabla\left|\nabla f * G_{r_{c}}\right|$, which has a $G_{r_{c}}$ limited range. For sufficient edge pull we take $\mu=0.2$.

\section{RESULTS}

Using the GVF field to guide the dot pattern initialized DDC, we can converge to the final contour. An example is given in Fig. 6. To quantify the performance of the algorithm, the automatic segmentations are compared to manual segmentation by a clinical expert. In comparing the automated segmentation to the manual segmentation, we can define the sensitivity and the accuracy [7] as

$$
\begin{aligned}
\text { sensitivity } & =T P /(T P+F N) \\
\text { accuracy } & =1-(F P+F N) /(T P+F N)
\end{aligned}
$$

in which TP is the number of true positive pixels (pixels of the automated segmentation that overlap with the manual one), $F N$ is the number of false negative pixels (pixels that are part of the manual segmentation but not of the automated one) and FP stands for the number of false positive pixels (pixels that are part of the automated segmentation but not of the manual one).

For the 4 datasets analyzed, the results are summarized in the table in Fig. 6.

For an image of $300 \times 400$ pixels the calculation time is about $1 \mathrm{~min}$ on a $4 \mathrm{~GB}$ RAM $2.53 \mathrm{GHz}$ machine. About $45 \mathrm{~s}$ of this time is needed for the Hessian dot evaluation.

\section{DISCUSSION}

Our preliminary results confirm the feasibility and accuracy of the method, although more extensive validation is necessary. However, if the granulation of the echographic images

\begin{tabular}{|c|c|c|c|}
\hline \multirow{3}{*}{ Final segmentation } & Image \# & Sens & Acc \\
\hline & 1 & 0.93 & 0.92 \\
\hline & 2 & 0.91 & 0.87 \\
\hline & 3 & 0.95 & 0.90 \\
\hline & 4 & 0.94 & 0.92 \\
\hline & Mean & 0.93 & 0.90 \\
\hline & Std & 0.015 & 0.022 \\
\hline & & \multicolumn{3}{|c}{} \\
\hline & &
\end{tabular}

Fig. 6. Final segmentation, evolved from the initial segmentation in Fig. 4 , and result table.

is clear enough, the granulation detector combined with the Hessian granulation evaluator extracts relevant granulation effectively. In this case, a proper granulation-based contour initialization can be made. If the initialization is near the relevant edges, the GVF field offers proper edge guiding to evolve the initial contour to the final contour.

Points of attention for future research include the dot detector size, which was not tuned to the image under investigation. A multiscale approach was tested, but it turned out that combining dot detectors of different sizes does not improve overall algorithm performance. Nevertheless it seems relevant to derive the optimal dot detector size from the image.

Concerning the edge guiding, it might be interesting to search for alternatives for calculating the GVF field from a binary edge map. This would permit avoiding the critical choice of a proper value for $\mu$ in (13).

\section{REFERENCES}

[1] J. Noble and D. Boukerroui, "Ultrasound image segmentation: a survey," IEEE Trans. Medical Imaging, vol. 25, no. 8, pp. 987-1010, 2006.

[2] A. Zaim, "FSM: A new finite sphere method for modeling 3D geometry of the prostate," IEEE ICIP proceedings, pp. 2956-2959, 2008.

[3] R. J. Eckersley, J. P. M. Sedelaar, M. J. K. Blomley, H. Wijkstra, N. M. deSouza, D. O. Cosgrove, and J. J. M. C. H. de la Rosette, "Quantitative microbubble enhanced transrectal ultrasound as a tool for monitoring hormonal treatment of prostate carcinoma," The Prostate, vol. 51, no. 4, pp. 256-267, 2002.

[4] M. K. Brawer, "Quantitative microvessel density: a staging and prognostic marker for human prostatic carcinoma," Cancer, vol. 78, no. 2, pp. 345-349, 1996.

[5] J. A. Siegal, E. Yu, and M. K. Brawer, "Topography of neovascularity in human prostate carcinoma," Cancer, vol. 75, no. 10, pp. 2545-2551, 1995.

[6] A. Zaim and J. Jankun, "An energy-based segmentation of prostate from ultrasound images using dot-pattern select cells," IEEE ICASSP proceedings, pp. 297-300, 2007.

[7] H. Ladak, F. Mao, Y. Wang, D. Downey, D. Steinman, and A. Fenster, "Prostate segmentation from 2d ultrasound images," IEEE EMBS proceedings, vol. 4, pp. 3188-3191, 2000.

[8] N. Petkov and W. T. Visser, "Modifications of centersurround, spot detection and dot-pattern selective operators," Technical report 2005-9-01, pp. 1-4, 2005. [Online]. Available: http://www.cs.rug.nl/ petkov/publications/2005cs-rep_dot-pat.pdf

[9] E. Franken, "Enhancement of crossing elongated structures in images," Ph.D. dissertation, Eindhoven University of Technology, Netherlands, 2008.

[10] C. Y. Xu and J. L. Prince, "Snakes, shapes, and gradient vector flow," IEEE Trans. Image Processing, vol. 7, no. 3, pp. 359-369, 1998.

[11] C. Xu and J. L. Prince, Handbook of Medical Imaging - Processing and Analysis. Academic press, ch. 10. 